\title{
Mechanische Feldschwächung einer Multi-Rotor-Permanentmagnet- Synchronmaschine
}

\author{
A. Brunner, R. Spießberger, M. Schrödl
}

In dieser Arbeit wird ein Konzept einer neuartigen mechanischen Feldschwächmethode am sogenannten elektrischen Planetenmotor präsentiert. Der elektrische Planetenmotor ist eine Kombination aus einer elektrischen Maschine mit mehreren Rotoren in einem gemeinsamen Stator und einem Getriebeteil, wobei die spezielle Anordnung des Getriebes zur mechanischen Feldschwächung genützt werden kann. Es wird die theoretische Grundlage dafür erarbeitet sowie die Funktionsweise anhand von Messungen an einem Prototyp demonstriert.

Schlüsselwörter: Planetenmotor; mechanische Feldschwächung; Multi-Rotor-PSM

\section{Mechanical field-weakening method of a multi-rotor permanent magnet synchronous machine.}

This work presents a concept of a novel mechanical field-weakening method developed for a multi-rotor permanent magnet synchronous machine called the Planetary Motor. The electric Planetary Motor combines an electric machine with multiple rotors in a common stator and a gear set, which can be utilized for mechanical field-weakening. This work presents the theoretical background as well as measurements on a prototype equipped with this field-weakening method.

Keywords: Planetary Motor; mechanical field-weakening; multi-rotor PMSM

Eingegangen am 21. Mai 2021, angenommen am 5. Juli 2021, online publiziert am 20. Juli 2021

(c) The Author(s) 2021

\section{Einleitung}

Permanentmagneterregte Synchronmaschinen werden in elektrischen Antrieben häufig mit hoher Drehzahl betrieben, um eine hohe Leistungsdichte zu erreichen. Traktionsantriebe benötigen typischerweise ein konstantes, hohes Drehmoment bei niedriger Drehzahl sowie eine konstante Leistung im hohen Drehzahlbereich, in dem die elektrische Maschine im Feldschwächbetrieb betrieben wird. Die erforderliche Drehzahlreduktion von der elektrischen Maschine zur Antriebsachse wird durch ein zusätzliches Getriebe erreicht. Bei hohen Drehzahlen kann die notwendige elektrische Feldschwächung ein Risiko im Fall eines Fehlers des Umrichters darstellen. Beim Ausfall der elektrischen Feldschwächung kann so die induzierte Spannung der Maschine die DC Spannung des Umrichters übersteigen [1-3]. Um dieses Risiko im Fehlerfall zu minimieren können entsprechende Maßnahmen sowohl in der Auslegung des Umrichters als auch der Maschine getroffen werden, oder alternativ eine mechanische Feldschwächmethode eingesetzt werden.

Für konventionelle Permanentmagneterregte Synchronmaschinen (PSM) wurden bereits einige Methoden zur mechanischen Feldschwächung entwickelt. Dabei war nicht nur die Risikominimierung im Fehlerfall eine Motivation, sondern auch die Erhöhung des Wirkungsgrads der Maschine [4]. Die Arbeit von El-Refaie [5] bietet eine umfangreiche Übersicht über Methoden zur Absicherung gegen diverse Fehlerfälle der PSM, wobei ein Teil dieser Methoden auch die mechanische Feldschwächung gegen überhöhte Klemmenspannungen umfasst. Im Kern dieser mechanischen Feldschwächvarianten steckt immer die Umlenkung des magnetischen Flusses, z.B. über bewegliche magnetische Rückschlussteile oder bewegliche Magne- te, sodass der Verkettungsfluss der Statorspulen gesenkt oder ausgelöscht wird.

Eine allgemein gefasste Arbeit ausschließlich zur Feldschwächung von Synchronmaschinen von Woehl-Bruhn et al. [6] zeigt eine Klassifizierung unterschiedlicher Feldschwächmethoden, in der auch Möglichkeiten zur mechanischen Feldschwächung berücksichtigt werden. Weiters wird in der Arbeit ein neuartiges Konzept vorgestellt, in dem ein vierpoliger Rotor mit zusätzlichen drehbar gelagerten Permanentmagneten ausgestattet wird, die durch ihre Verdrehung eine Schwächung des Hauptflusses ermöglichen. Eine weitere Möglichkeit zeigt [7], wo durch eine axiale Verschiebung der gesamte Rotor kontinuierlich aus der Statorbohrung geschoben wird, bis der gewünschte Feldschwächgrad erreicht ist.

Auch für Axialflussmaschinen wurden Methoden zur mechanischen Feldschwächung entwickelt, wobei hier häufig auf zentrifugalkraftbasierte Mechanismen zurückgegriffen wurde. Beispielsweise wird in [8] mit steigender Drehzahl durch die Zentrifugalkraft der Luftspalt so vergrößert, dass die induzierte Spannung mit steigender Drehzahl konstant bleibt. Ein weiteres Patent [9] beschreibt eine Axialflussmaschine, bei der durch radial beweglich gelagerte Permanentmagnete im Rotor die Überdeckung mit der Statorwicklung au-

Brunner, Andreas, Institut für Energiesysteme und Elektrische Antriebe, Technische Universität Wien, Gußhausstraße 25/370-02, 1040 Wien, Österreich (E-Mail: andreas.brunner@tuwien.ac.at); Spießberger, Richard, Institut für Energiesysteme und Elektrische Antriebe, Technische Universität Wien, Wien, Österreich; Schrödl, Manfred, Institut für Energiesysteme und Elektrische Antriebe, Technische Universität Wien, Wien, Österreich 


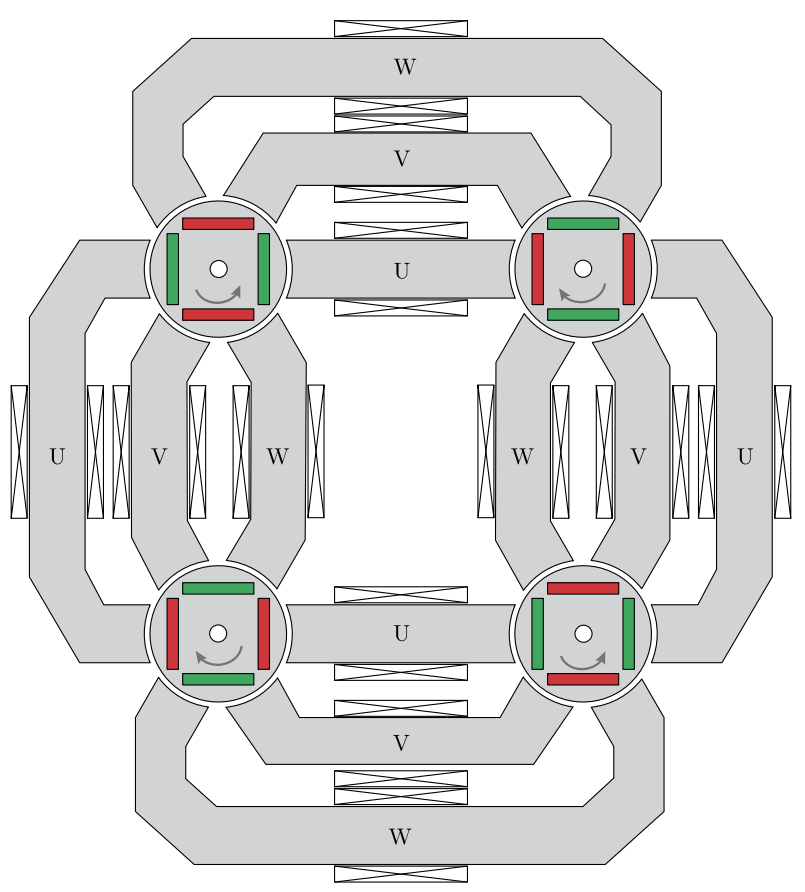

Abb. 1. Schematische Struktur des Planetenmotors in einer Variante mit vier vierpoligen Rotoren und zwölf Statorwicklungen

tomatisch durch die steigende Zentrifugalkraft angepasst wird und auch so eine Schwächung des Verkettungsflusses eingestellt wird.

Die besondere Struktur des elektrischen Planetenmotors als MultiRotor-Maschine ermöglicht nun eine neue Art der mechanischen Feldschwächung, bei der der Verkettungsfluss der Statorspulen über eine Verdrehung der Rotoren aus ihrer Normalposition während des Betriebs variiert und nur durch einen einzelnen zusätzlichen Aktuator gesteuert werden kann. Nach der Einteilung von [6] kann diese Methode der Kategorie „Änderung des magnetischen Widerstands" über "Verstellung der Aktivteile" zugeordnet werden. Dazu wird zuerst in Abschn. 2 die Struktur und Funktionsweise des Planetenmotors zusammengefasst, bevor in Abschn. 3 die Feldschwächmethode hergeleitet wird. Weiters folgen Ergebnisse aus Simulationen in Abschn. 4 sowie Messergebnisse des realisierten Prototyps in Abschn. 5.

\section{Der elektrische Planetenmotor}

Der elektrische Planetenmotor wurde erstmals 2017 von Manfred Schrödl vorgestellt [10]. Diese erste Variante des Planetenmotors basiert auf vier zweipoligen Permanentmagnetrotoren und diente als Machbarkeitsnachweis für diese Maschinenstruktur. Der Planetenmotor ist aber nicht auf zweipolige Rotoren oder genau diese Statorstruktur beschränkt, sondern es sind auch andere Varianten möglich. Beispielsweise können sechs oder acht Rotoren eingesetzt werden, sowohl in zweipoliger als auch vierpoliger Ausführung. Abbildung 21 zeigt ein Konzept mit vierpoligen Rotoren und nur sechs Statorspulen, das einen kompakten Aufbau inklusive Kühlelementen erlaubt. Die verzahnten Rotorwellen stellen direkt den Getriebeeingang dar und greifen paarweise in ein Sonnenund Hohlrad, welche widerrum mechanisch verbunden sind und so die Leistung aller Rotoren auf eine gemeinsame Abtriebswelle summieren. Die notwendige räumliche Ausdehnung der Getriebeeinheit erlaubt hohe Übersetzungen in der Größenordnung von 10:1. Die Ähnlichkeit zu einem Planetengetriebe war auch der Namensgeber dieser Maschine. Deshalb wurde die Nomenklatur mit

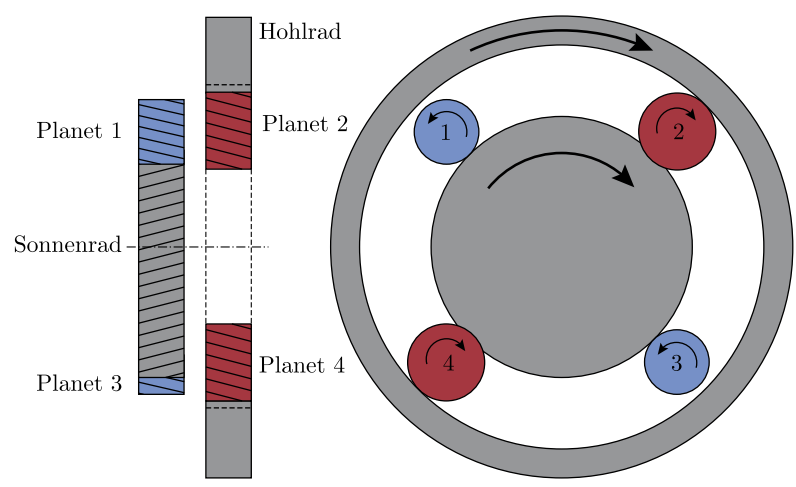

Abb. 2. Modifiziertes Planetengetriebe zur Kopplung der vier Rotoren. Rotorwellen und mechanische Verbindung von Sonnen- und Hohlrad nicht dargestellt

Sonnen-, Hohl- und Planetenrädern übernommen, obwohl diese streng genommen nicht korrekt ist. In dieser Arbeit wird eine Variante des Planetenmotors beschrieben, in welcher vierpolige Rotoren mit vergrabenen Permanentmagneten eingesetzt werden und jeder der vier Rotoren sechs umliegende Statorzähne besitzt, siehe Abb. 1.

Jeder der zwölf Statorschenkel trägt eine konzentrierte Spule, welche zu einer im Stern verschaltenen, dreisträngigen Drehstromwicklung führen. Für die Herleitung dieser Struktur sei auf [11] verwiesen. Diese Drehstromwicklung wird von einem einzigen konventionellen Umrichter gespeist und erzeugt vier synchron rotierende Drehfelder. Die Drehrichtung wird durch die Strangzuordnung der einzelnen Spulen festgelegt, sodass die Rotoren jeweils gegenläufig rotieren. Die Rotorachsen liegen exakt auf einem Rechteck, was eine axiale Verschiebung von Sonnen- und Hohlradebene notwendig macht, um eine Überschneidung dieser beiden Stufen zu vermeiden, siehe Abb. 2. Das Sonnen- und Hohlrad sind schließlich über ein (nicht dargestelltes) Verbindungsstück mit der Hauptabtriebswelle gekoppelt. Diese mechanische Kopplung aller Rotoren wird nun zur mechanischen Feldschwächung genutzt.

\section{Mechanische Feldschwächung des Planetenmotors}

\subsection{Ersatzmodell des Planetenmotors}

Zur mathematischen Beschreibung der Feldschwächmethode mittels Raumzeigerrechnung kann ein vereinfachtes Ersatzmodell des Planetenmotors mit nur zwei Rotoren herangezogen werden, siehe Abb. 3. Aufgrund der Symmetrie der Maschinenstruktur kann dieses Modell für eine beliebige, gerade Anzahl von Rotoren angewendet werden.

\subsection{Normalbetrieb}

Zur Herleitung wird die übliche Raumzeigerrechnung mit den für eine PSM gängigen statorfesten $\alpha \beta$ - sowie rotorfesten $d q$ Koordinatensystemen verwendet. In erster Näherung kann angenommen werden, dass die Permeabilität des Stator- und Rotorblechs unendlich ist und damit Sättigungseffekte ausgenommen sind. Weiters wird die Maschine im stromlosen Zustand analysiert, wodurch die Raumzeiger der Verkettungsflüsse nur eine Folge der Permanentmagnete in den Rotoren sind und damit unmittelbar die Richtung der $d$-Achsen der Rotoren repräsentieren. Die Einschränkung auf stromlose Wicklungen ist aber keine notwendige Voraussetzung für 


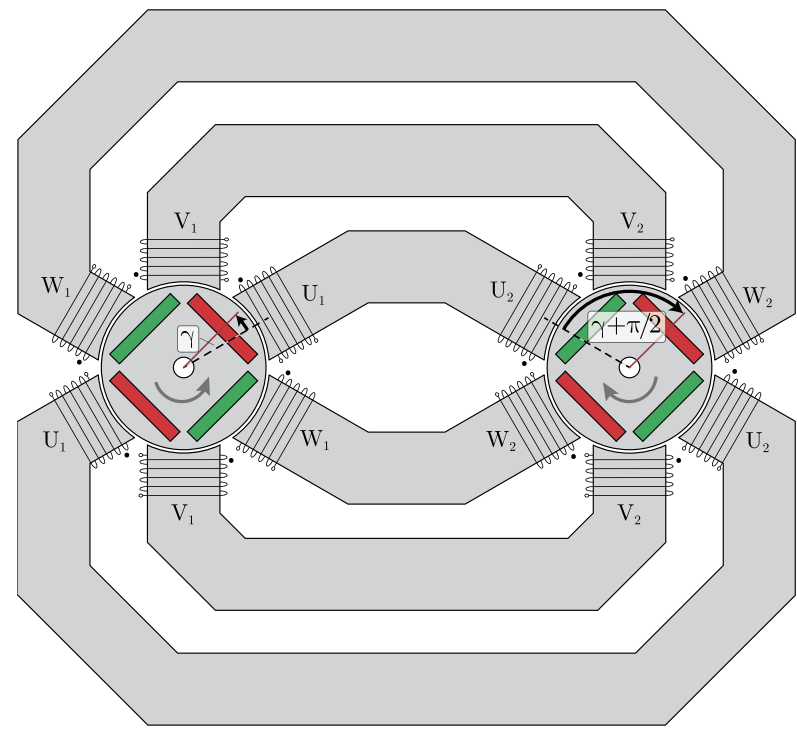

Abb. 3. Lage der Rotoren im Normalbetrieb, beide um den beliebigen Winkel $\gamma$ in ihren positiven Drehrichtungen aus der Initialposition gedreht. Zweiter Rotor gegenüber dem ersten Rotor um $180^{\circ}$ (elektr.) verdreht
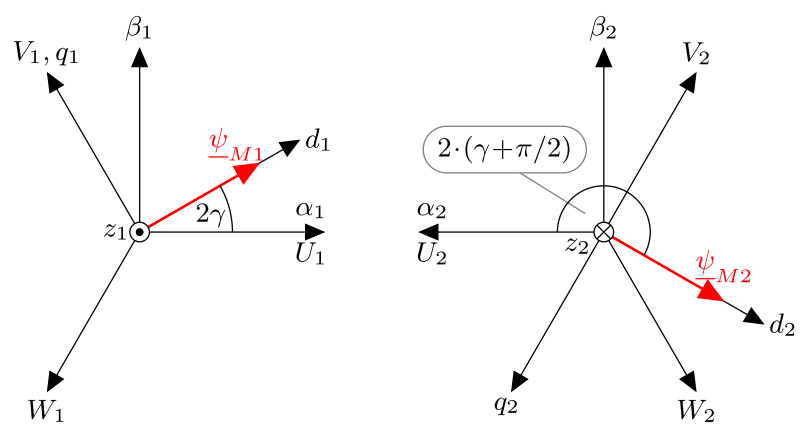

Abb. 4. Raumzeigerdiagramme der getrennt betrachteten Maschinen (links und rechts passend zu Abb. 3)

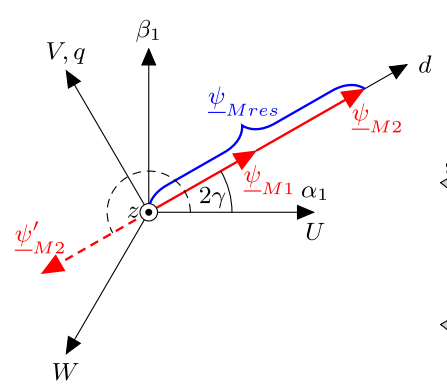

(a) Ersatz-Raumzeiger

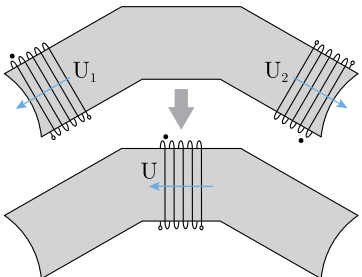

(b) Spulen am selben Statorteil
Abb. 5. Ersatz-Raumzeiger im Normalbetrieb

die Anwendung dieser Feldschwächmethode. Ordnet man nun jedem Rotor ein eigenes, dreisträngiges Wicklungssystem zu $\left(U_{1}, V_{1}\right.$, $W_{1}$ bzw. $U_{2}, V_{2}, W_{2}$, Abb. 3) und behandelt diese als voneinander unabhängig, können in klassischer Weise zwei Raumzeigerdiagramme gezeichnet werden, siehe Abb. 4. Dabei ist die unterschiedli-

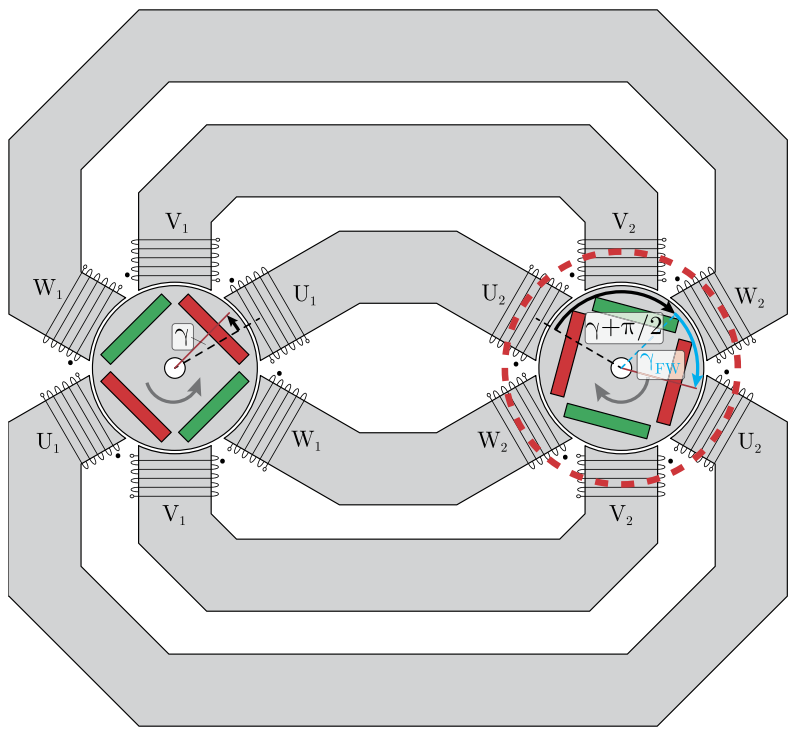

Abb. 6. Lage der Rotoren im Feldschwächbetrieb. Zweiter Rotor in Bezug auf Abb. 3 um zusätzlichen Winkel $\gamma_{F W}=60^{\circ}$ (mech.) gedreht

che Orientierung der z-Achsen der beiden Diagramme zu beachten.

Nun kann die Maschine von außen (aus Sicht des Umrichters an den Klemmen) durch eine dreisträngige Ersatzmaschine beschrieben werden, in der es nur einen äquivalenten rotierenden Verkettungsflussraumzeiger gibt (bildlich gesprochen also nur einen Rotor). Betrachtet man die beiden Spulen eines gemeinsamen Statorteils, so sind diese näherungsweise mit dem gleichen magnetischen Fluss verkettet, und können so unter Berücksichtigung der Wicklungsorientierung zu einer Spule zusammengefasst werden (Abb. 5b). Als Bezugssystem wird dazu das $\alpha_{1} \beta_{1}$-Koordinatensystem gewählt. Transformiert man den Verkettungsflussraumzeiger $\underline{\psi}_{M 2}$ aus dem $\alpha_{2} \beta_{2}$ - in das $\alpha_{1} \beta_{1}$-Koordinatensystem $\left(\psi_{M 2}^{\prime}\right)$, muss dieser noch aufgrund der Spulenorienterung am Statorschenkel um $180^{\circ}$ gedreht werden. Der Verkettungsflussraumzeiger $\underline{\psi}_{\text {Mres }}$ ergibt sich nun im $\alpha_{1} \beta_{1}$-Koordinatensystem direkt aus der Superposition der beiden Teilverkettungsflüsse $\psi_{M 1}$ und $\psi_{M 2}$ (Abb. 5a). Die Gleichungen (1)-(5) zeigen, dass dieser in die selbe Richtung wie $\underline{\psi}_{M 1}$ zeigt, aber im Betrag verdoppelt wurde. Gedanklich könnte man dieses Experiment auch auf weitere Rotoren fortsetzen.

$$
\begin{aligned}
\underline{\psi}_{M 1} & =\underline{\psi}_{\alpha \beta, 1}=\psi_{M 1} e^{j 2 \gamma} \\
\underline{\psi}_{M 2} & =\underline{\psi}_{\alpha \beta, 2}=\psi_{M 2} \mathrm{e}^{j(2 \gamma+\pi)}=-\psi_{M 2} \mathrm{e}^{j 2 \gamma} \\
\psi_{M 1} & =\psi_{M 2}=\psi_{M} \\
\underline{\psi}_{\alpha \beta} & =\underline{\psi}_{\text {Mres }}=\underline{\psi}_{\alpha \beta, 1}-\underline{\psi}_{\alpha \beta, 2}=2 \psi_{M} \mathrm{e}^{j 2 \gamma} \\
\left|\underline{\psi}_{\alpha \beta}\right| & =\left|\underline{\psi}_{\text {Mres }}\right|=2 \psi_{M}
\end{aligned}
$$

\subsection{Feldschwächbetrieb}

Verdreht man ausgehend von diesen Rotorpositionen beispielsweise nur Rotor 2 um einen Winkel $\gamma_{F W}$ (Abb. 6), verändern sich die Zeigerdiagramme entsprechend Abb. 7 und 8. Bildet man nun wieder den Betrag des resultierenden Verkettungsflussraumzeigers (9), so erkennt man, dass sich dieser proportional $\cos \left(\gamma_{F W}\right)$ verringert. Bei Betrachtung von Abb. 8 bzw. Gl. (8) fällt auf, dass sich nicht nur der Betrag des resultierenden Verkettungsflusses ändert, sondern auch 

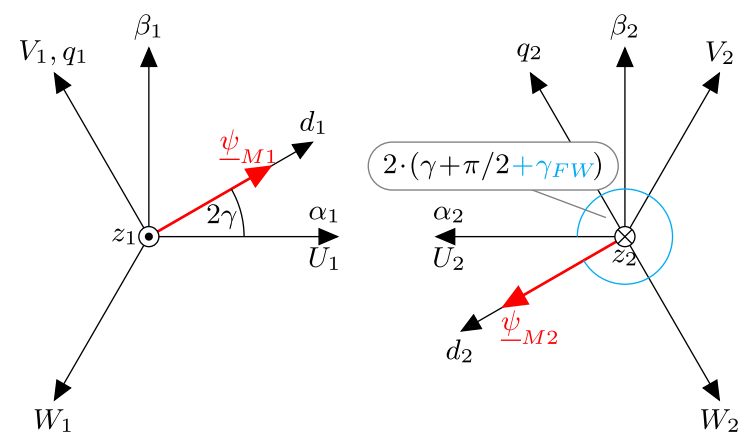

Abb. 7. Raumzeigerdiagramme der getrennt betrachteten Maschinen im Feldschwächbetrieb (links und rechts passend zu Abb. 6)

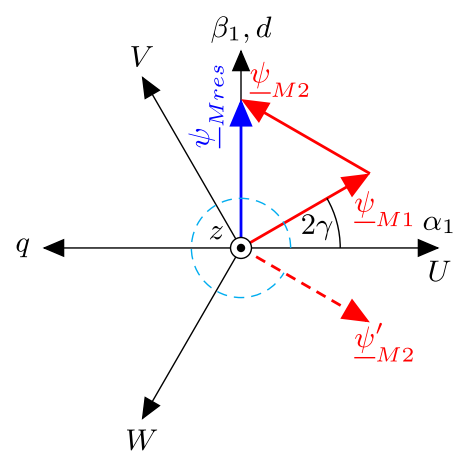

Abb. 8. Ersatz-Raumzeiger im Feldschwächbetrieb

dessen Winkel zur $\alpha_{1}$-Achse.

$$
\begin{aligned}
\underline{\psi}_{\alpha \beta, 1} & =\psi_{M} e^{j 2 \gamma} \\
\underline{\psi}_{\alpha \beta, 2} & =\psi_{M} e^{j\left(2 \gamma+\pi+2 \gamma_{F W}\right)}=-\psi_{M} e^{j\left(2 \gamma+2 \gamma_{F W}\right)} \\
\underline{\psi}_{M r e s} & =\underline{\psi}_{\alpha \beta, 1}-\underline{\psi}_{\alpha \beta, 2}=\psi_{M} e^{j 2 \gamma} \cdot\left(1+e^{j 2 \gamma_{F W}}\right) \\
\left|\underline{\psi}_{\alpha \beta}\right| & =\left|\underline{\psi}_{M r e s}\right|=\psi_{M} \cdot \sqrt{2+2 \cos \left(2 \gamma_{F W}\right)} \\
& =\psi_{M} \cdot \sqrt{4 \cos ^{2}\left(\gamma_{F W}\right)}=2 \psi_{M} \cos \left(\gamma_{F W}\right)
\end{aligned}
$$

Setzt man diese Variante der mechanischen Feldschwächung mit einer feldorientierten Regelung ein, muss diese Drehung der resultierenden $d$-Achse berücksichtigt werden. Falls der Winkel $\gamma_{F W}$ bekannt ist, kann diese Verdrehung der $d$-Achse mit GI. (8) ermittelt werden.

Alternativ könnte man die Verdrehung um $\gamma_{F W}$ nicht nur auf einem Rotor einprägen, sondern bei beiden Rotoren den Winkel $\gamma_{F W} / 2$, da nur die relative Ausrichtung der Rotoren zueinander ausschlaggebend ist. Dabei ist zu beachten, dass der zweite Rotor wie bisher in seiner positiven Drehrichtung (grauer Pfeil), der erste Rotor aber entgegen seiner positiven Drehrichtung um den zusätzlichen Winkel $\gamma_{F W} / 2$ gedreht wird:

$$
\begin{aligned}
& \underline{\psi}_{\alpha \beta, 1}=\psi_{M} e^{i\left(2 \gamma-\gamma_{F W}\right)} \\
& \underline{\psi}_{\alpha \beta, 2}=\psi_{M} e^{j\left(2 \gamma+\pi+\gamma_{F W}\right)}=-\psi_{M} e^{j\left(2 \gamma+\gamma_{F W}\right)} \\
& \underline{\psi}_{\text {Mres }}=\underline{\psi}_{\alpha \beta, 1}-\underline{\psi}_{\alpha \beta, 2}=2 \psi_{M} \cos \left(\gamma_{F W}\right) \cdot e^{j 2 \gamma}
\end{aligned}
$$

Wie die Abb. 9 und 10 sowie Gl. (12) zeigen, ergibt sich in dieser Variante derselbe Betrag des Verkettungsflusses $\underline{\psi}_{\text {Mres' }}$ aber mit
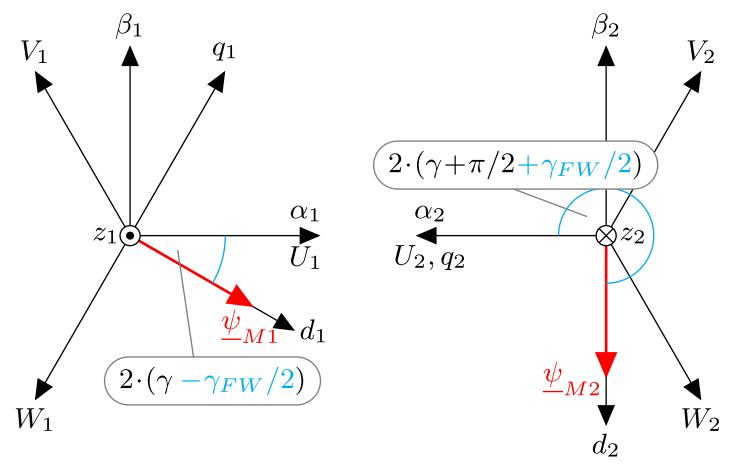

Abb. 9. Raumzeigerdiagramme der getrennt betrachteten Maschinen im Feldschwächbetrieb, Verdrehung beider Rotoren um $\gamma_{F W} / 2$

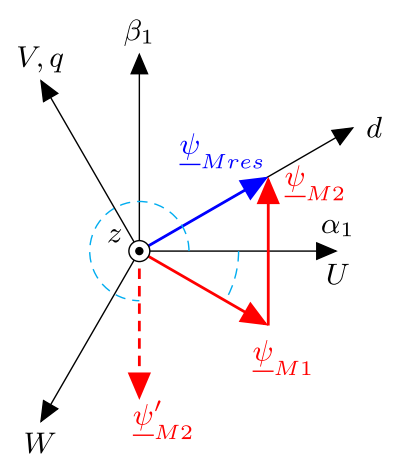

Abb. 10. Ersatz-Raumzeiger im Feldschwächbetrieb, Verdrehung beider Rotoren um $\gamma_{F W} / 2$

unveränderter Orientierung der resultierenden $d$-Achse im Vergleich zum Normalbetrieb.

\section{Simulationsergebnisse}

Dieses vereinfachte analytische Modell wurde mit einer 2D-FEM Simulation verifiziert. Hierbei wurde allerdings nicht das Ersatzmodell mit zwei Rotoren simuliert, sondern das vollständige Modell mit vier (ungeschrägten) Rotoren entsprechend dem später realisierten Prototyp.

\subsection{Induzierte Spannung}

Um in der Simulation verschiedene Feldschwächgrade vergleichen zu können, wurde die induzierte Außenleiterspannung im Leerlauf bei einer konstanten Drehzahl ermittelt. Die Herleitung in Abschn. 3 berücksichtigt nur die Grundschwingung, also ist zu erwarten, dass mit zunehmenden Feldschwächgrad der Anteil der Oberschwingungen in der Spannung zunimmt, analog zur klassischen elektrischen Feldschwächung. Abbildung 11 zeigt repräsentativ einige Spannungsverläufe für unterschiedliche Feldschwächgrade. Auffallend ist die ideal ausgelöschte Spannung bei $\gamma_{F W}=90^{\circ}$. Hier schließt sich im Simulationsmodell der gesamte magnetische Fluss zufolge der Permanentmagnete als Streufluss in der Maschine, ohne dass es eine resultierende Verkettung mit den Statorwicklungen gibt. Die hochfrequenten Anteile der induzierten Spannung sind auf dünne, zwischen den Statorzähnen liegende Blechstege zurückzuführen, welche zum Zeitpunkt der Planung des Prototyps als mechanisch notwendig angesehen wurden. Diese sind zwar größtenteils magnetisch gesättigt und damit kaum merkbar, aber sie führen an bestimmten Rotorpositionen durch kurzzeitige Entsättigung zu diesen hochfrequenten Spannungsspitzen. 


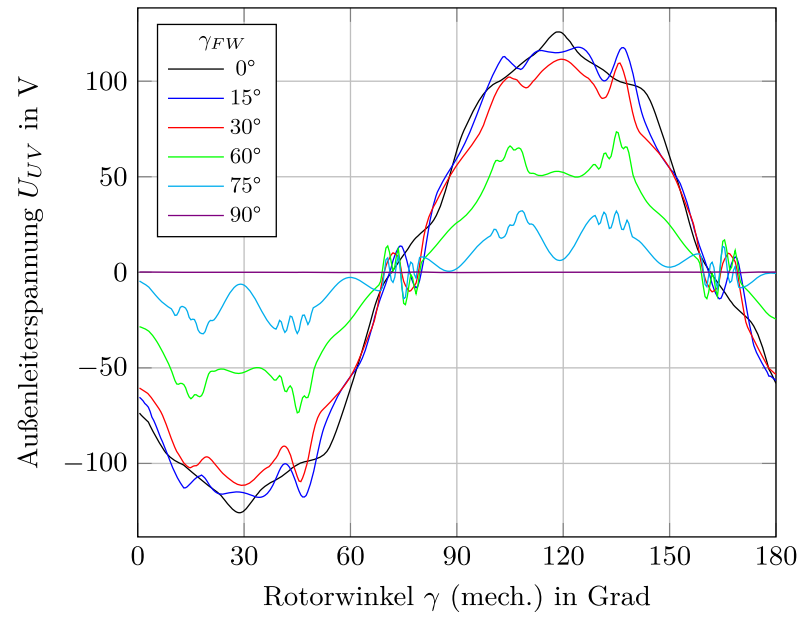

Abb. 11. Simulierte Leerlaufspannung bei $n=0,5$ und Variation des Feldschwächwinkels $\gamma_{F W}$

Bei näherer Betrachtung im Frequenzbereich (Abb. 12) ist ersichtlich, dass die Grundschwingung sehr gut dem analytischen Modell nach (12) folgt, weil deren Amplitude bei $\gamma_{F W}=60^{\circ}$ in guter Näherung die Hälfte der Amplitude bei $\gamma_{F W}=0^{\circ}$ ist. Weiters fällt auf, dass es über alle Feldschwächgrade hinweg einen kleinen, aber wahrnehmbaren Anteil der 3. Harmonischen in der Außenleiterspannung gibt. Diese tritt ursprünglich in den Strangspannungen zufolge kleiner Sättigungsbereiche im Blech auf, welche sich allerdings bei der Bildung der Außenleiterspannung aufheben sollte. Dies ist hier aufgrund einer Abweichung von der idealen Strangsymmetrie nicht gegeben, sodass ein kleiner Anteil der 3. Harmonischen (und deren Vielfache) in den Außenleitergrößen übrig bleibt. Die Stränge $U$ und $W$ wechseln jeweils zwischen innen- und außenliegendem Statorschenkel (siehe Abb. 1), sodass sie über die gesamte Maschine wieder symmetrisch sind, aber es dennoch eine Unsymmetrie gegenüber dem Strang $\vee$ gibt. Eine weitere theoretische Ursache für diesen Effekt ist eine von $2 \pi / 3$ abweichende Phasendifferenz zwischen den Strangspannungen, welche aber aufgrund der ideal festgelegten Initialposition der Rotoren und deren eingeprägter Drehzahl in der Simulation ausgeschlossen werden kann. Betrachtet man einen einzelnen Rotor und dessen umliegende Statorzähne, kann man die Statorwicklung über die Lochzahl

$$
q=\frac{Q}{2 p \cdot m}=\frac{6}{4 \cdot 3}=\frac{1}{2}
$$

als Bruchlochwicklung beschreiben. Die zu erwartenden Ordnungszahlen 5, 7, 11, 13, etc. der Oberschwingungen der Spannung ([12]) treten auch in der berechneten Außenleiterspannung auf. Das bestätigt die Theorie, dass sich die Maschine von den Klemmen betrachtet wie eine gewöhnliche PSM mit einem Rotor verhält. Wie erwartet erkennt man mit zunehmendem Feldschwächgrad einen höheren Anteil der Oberschwingungen in der Spannung, da die Feldschwächung nur auf die 1. Harmonische wirkt. Dies ist auch über den steigenden Klirrfaktor der Außenleiterspannung

$$
k_{U}=\frac{\sqrt{U_{2}^{2}+U_{3}^{2}+U_{4}^{2}+\cdots}}{U}
$$

mit $U_{v}$ als Effektivwert der Oberschwingung mit der Ordnungszahl $v$ und $U$ als Effektivwert der Wechselgröße ersichtlich, siehe Tab. 1.

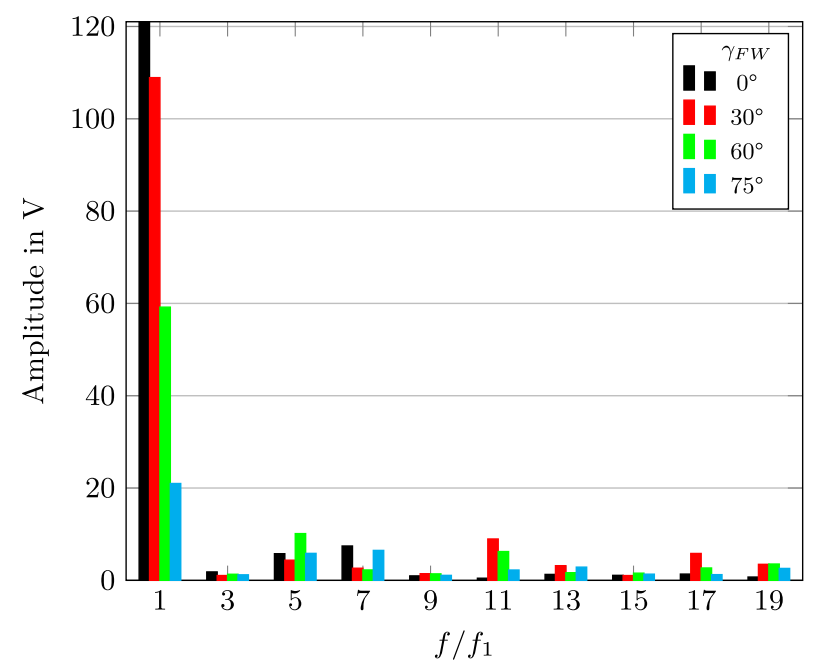

Abb. 12. Harmonische Analyse der Leerlauf-Außenleiterspannung $U_{U V}$ bei $n=0,5$ und verschiedenem $\gamma_{F W}$

Tab. 1. Klirrfaktor (THD) der Spannung $\boldsymbol{U}_{U V}$ bei $\boldsymbol{n}=\mathbf{0 , 5}$

\begin{tabular}{llllll}
\hline$\gamma_{F W}$ in $^{\circ}$ & 0 & 15 & 30 & 60 & 75 \\
\hline$k_{U}$ in $\%$ & 8.2 & 12.4 & 12.6 & 23.1 & 50.9 \\
\hline
\end{tabular}

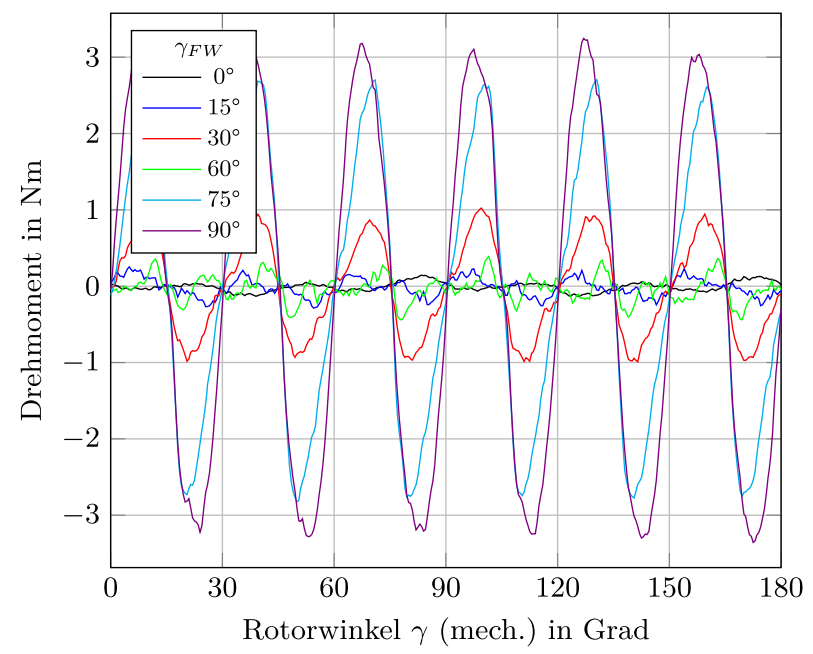

Abb. 13. Simuliertes elektromagnetisches Rastmoment (Summe aller Rotoren) im Leerlauf bei verschiedenem Feldschwächgrad

\subsection{Rastmoment}

Ein weiterer Aspekt, der in der Simulation untersucht wurde, war die Auswirkung des Feldschwächgrads auf das Rastmoment der Maschine. Die Rotoren sind im nicht-feldgeschwächten Zustand entsprechend ihrem Energieminimum im verkoppelten magnetischen Kreis ausgerichtet. Dreht man diese gedanklich nun aus dieser Ruhelage heraus, ist es leicht vorstellbar, dass ein zur Ausgangslage rückstellendes Drehmoment auftreten wird. Eine harmonische Analyse des Rastmomentverlaufs bei unterschiedlichen Feldschwächgraden im Leerlauf aus Abb. 13 zeigt eine dominante 6. und 12. harmonische Schwingung. Dieses Ergebnis war aus den dominanten 5. und 7. sowie 11. und 13. Harmonischen der Spannung zu erwarten. Ein interessanter Punkt ist die Tatsache, dass es in der Umge- 


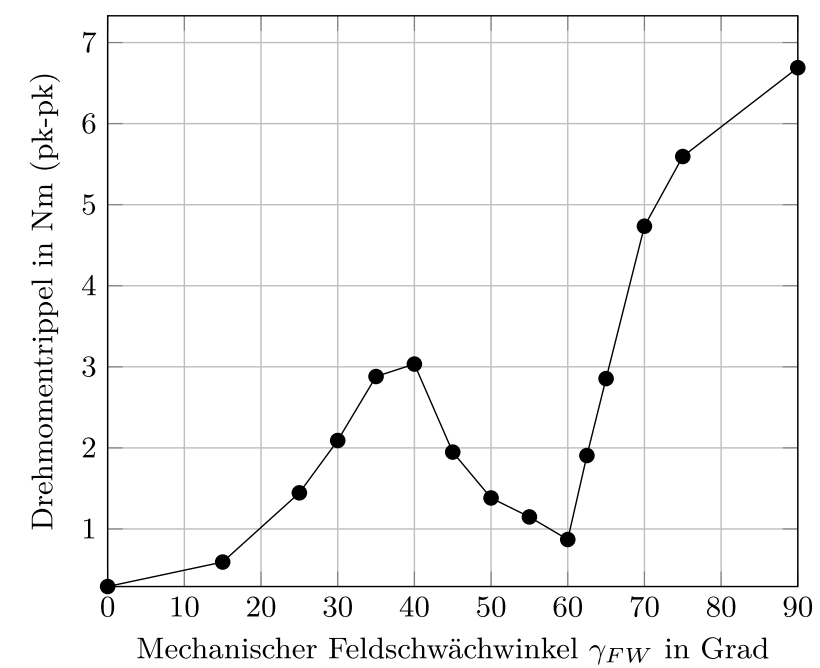

Abb. 14. Spitze-Spitze Rastmomentrippel bei verschiedenem Feldschwächgrad

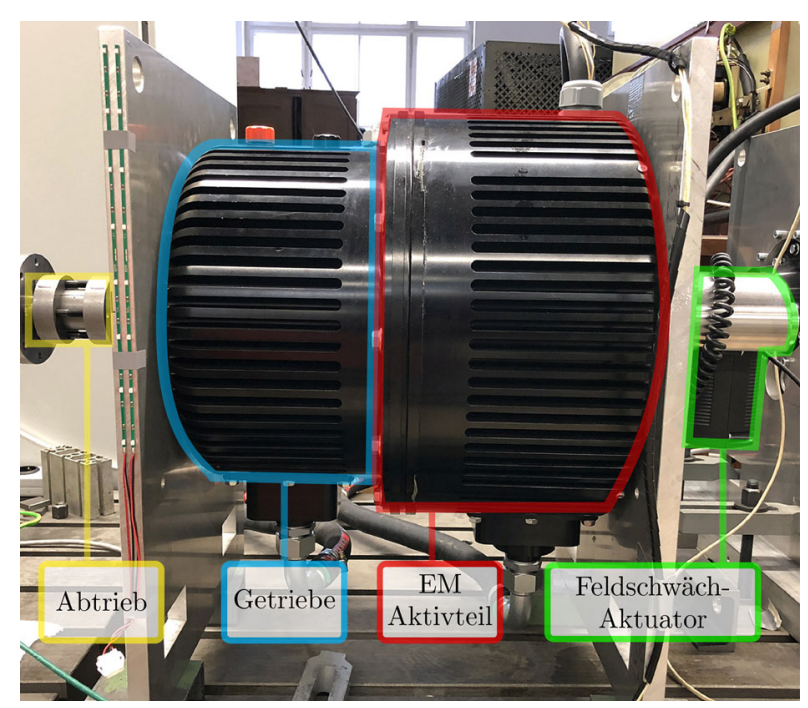

Abb. 15. Planetenmotor am Prüfstand

bung von $\gamma_{F W}=60^{\circ}$ zu einem starken Einbruch des Drehmomentrippels kommt. In der Nähe dieses Punktes heben sich die Einzelrotorrastmomente durch ihre Phasendifferenz teilweise auf, sodass ein insgesamt geringerer Drehmomentrippel in der Summe aller Rotoren entsteht, siehe Abb. 14. Bei maximaler Feldschwächung (bzw. auslöschung) stehen sich zwei Nord- bzw. Südpole benachbarter Rotoren direkt gegenüber, dabei tritt das größte Rastmoment auf. Besonders wenn die Feldschwächung unter Last verwendet wird, sollte dieser erhöhte Drehmomentrippel für die Auslegung des Getriebes berücksichtigt werden, da es auch einen Einfluss auf die Vibrationsund Geräuschentwicklung der Maschine hat. Wird diese Methode als Schutzsystem im Fehlerfall eingesetzt, spielt Letzteres aber eine untergeordnete Rolle.

\section{Messergebnisse}

Diese Feldschwächmethode wurde in einem Prototyp implementiert und am Prüfstand getestet. Die Nenndaten sind in Tab. 2 zusammengefasst. Für den Vergleich zur Simulation wurde der gleiche Betriebszustand bei konstanter, eingeprägter Drehzahl im Leerlauf gewählt.

\section{Tab. 2. Daten des Prototyps}

\begin{tabular}{ll}
\hline Nenndrehzahl Abtriebswelle & $850 \mathrm{~min}^{-1}$ \\
Nenndrehmoment Abtriebswelle & $480 \mathrm{Nm}$ \\
Übersetzung (1-stufig) & $1: 10$ \\
Rotorpole & 4 \\
Rotordurchmesser & $79 \mathrm{~mm}$ \\
Anzahl Rotoren & 4 \\
Eisen-Aktivlänge & $100 \mathrm{~mm}$ \\
\hline
\end{tabular}

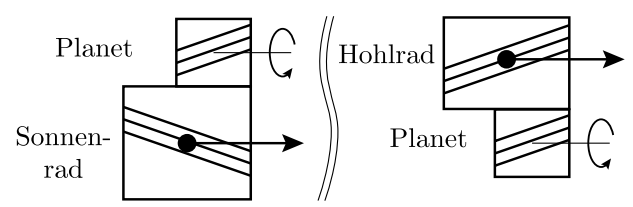

Abb. 16. Gleichzeitige Verschiebung von Sonnen- und Hohlrad zur Verdrehung der Rotoren

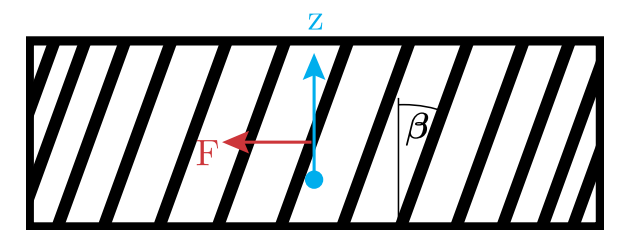

Abb. 17. Verdrehung der Rotoren durch axiale Verschiebung des schrägverzahnten Zahnrads

Die Maschine besteht im Wesentlichen aus dem modifizierten Planetengetriebe, in welchem das Sonnenrad und Hohlrad in zwei axial versetzten Ebenen platziert sind, dem elektromagnetischen Aktivteil sowie einem Feldschwächaktuator, siehe Abb. 15. Dieser Linearmotor bewegt die Sonnen- und Hohlradeinheit in axialer Richtung über eine Welle, die durch das Innere der Maschine verläuft, und stellt so unter Verwendung der schrägverzahnten Zahnräder synchron die Verdrehung aller vier Rotoren um den Feldschwächwinkel $\gamma_{F W} / 2$ ein, siehe Abb. 16. Bewegt man gedanklich den blauen Eingriffspunkt in Abb. 17 entlang der positiven Richtung der z-Achse, dreht sich das Zahnrad nach links. Kennt man den Schrägungswinkel $\beta$, kann über diese lineare Bewegung der Verdrehungswinkel berechnet werden. Die Konstruktion wurde für die Aktuierung unter Last bei laufender Maschine entsprechend ausgelegt.

\subsection{Induzierte Spannung}

Abbildung 18 zeigt die induzierte Leerlaufspannung im nichtfeldgeschwächten Zustand. Die Amplitude der Grundwelle der gemessenen Spannung ist zwar nur $2 \%$ geringer als bei der simulierten, allerdings weicht die Form (und damit der Oberschwingungsgehalt) doch deutlich erkennbar davon ab. Dieser Unterschied ist hauptsächlich darin begründet, dass die Nullpositionen der eingebauten Rotoren von der Idealausrichtung in der Simulation verschieden sind. Die in Abschn. 4.1 erwähnten Blechstege zwischen den Statorzähnen haben einen großen Einfluss auf die Lage und Gestalt der hochfrequenten Spannungsspitzen. Dieses sensitive Verhalten in Bezug auf die Nullposition der Rotoren konnte auch in weiteren Iterationen des Simulationsmodells nachgewiesen werden.

In Abb. 19 bestätigt sich das bekannte Verhalten der mechanischen Feldschwächung aus der Simulation. Mit zunehmendem Winkel $\gamma_{F W}$ wird der Betrag der induzierten Spannung geringer, und 


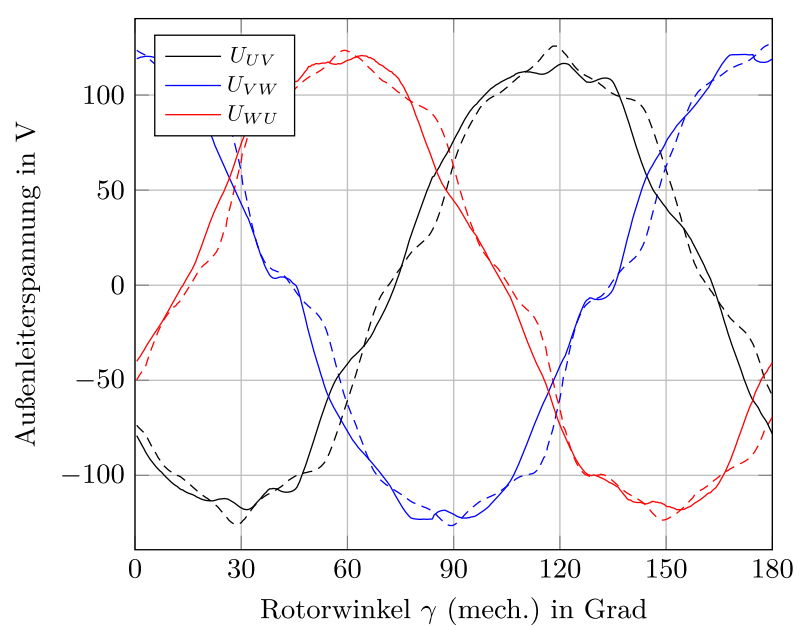

Abb. 18. Gemessene Leerlaufspannung bei $n=0,5$ (simulierte Spannung strichliert dargestellt)

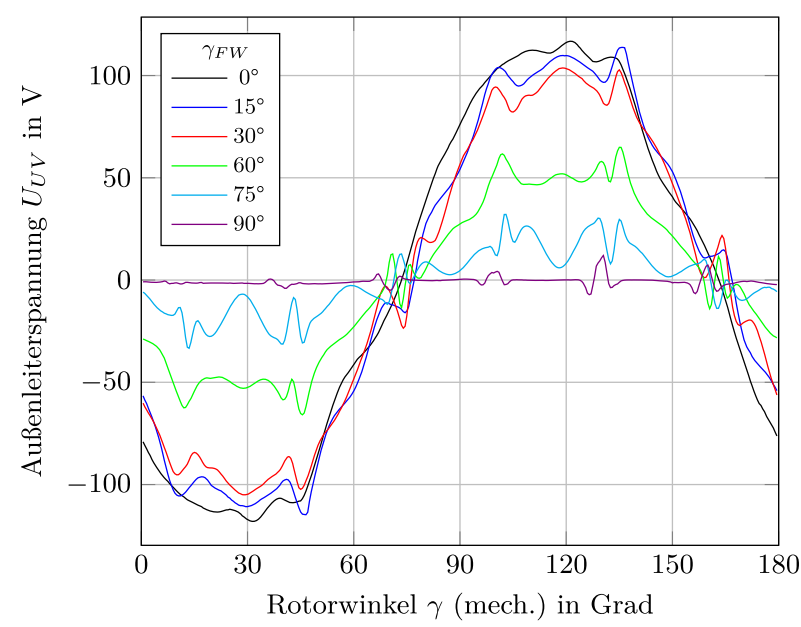

Abb. 19. Gemessene Leerlaufspannung bei $n=0,5$ und verschiedenem $\gamma_{F W}$

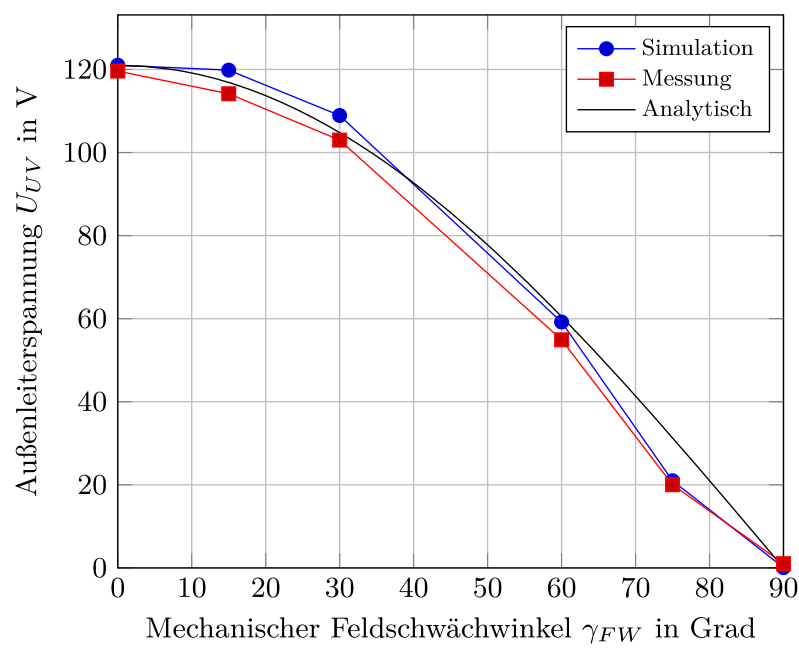

Abb. 20. Spitzenwert der Grundschwingung von $U_{U V}$ bei $n=0,5$

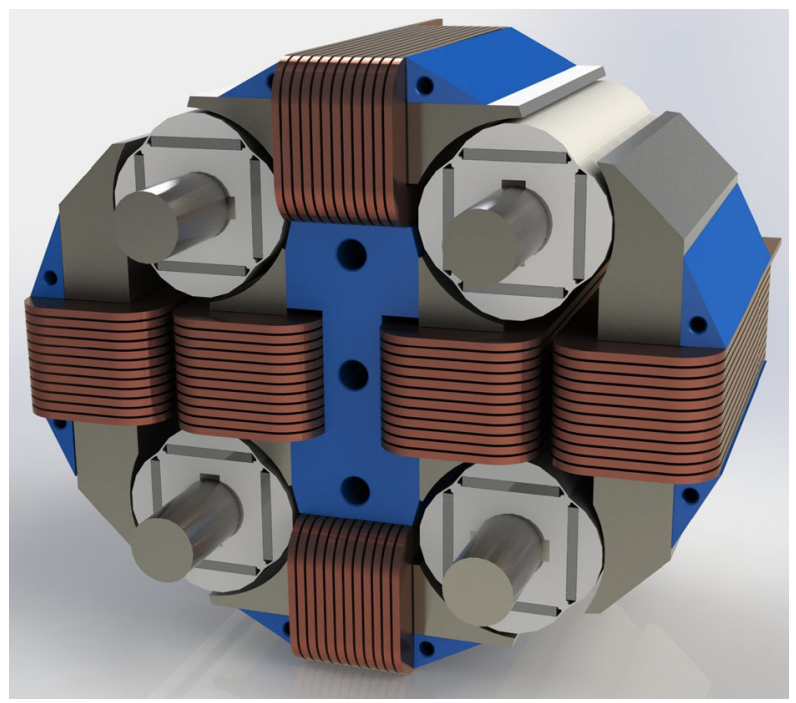

Abb. 21. Kompakter Modellentwurf eines Planetenmotors mit sechs Statorspulen, vierpoligen Rotoren und Kühlelementen

der Oberschwingungsanteil steigt. Die Amplituden der Außenleiterspannung aus Messung und Simulation zeigen in Abb. 20 eine gute Übereinstimmung mit dem cos-förmigen Verlauf aus dem analytischen Modell.

\subsection{Stationäres Drehmoment}

Das elektromagnetische Drehmoment lässt sich über die bekannte normierte Gleichung einer achsigen PSM im dq-Koordinatensystem über

$$
m=\psi_{M} i_{q}+i_{d} i_{q}\left(l_{d}-l_{q}\right)
$$

beschreiben, wobei $I_{d}$ und $l_{q}$ die normierten Statorinduktivitäten in Richtung $d-$ und $q$-Achse sind, und analog dazu $i_{d}$ und $i_{q}$ die Komponenten des normierten Statorstromraumzeigers. Für den feldgeschwächten Fall ist $\psi_{M}$ durch den geschwächten Betrag aus (12) zu ersetzen:

$$
m=\psi_{M}^{\prime} \cos \left(\gamma_{F W}\right) i_{q}+i_{d} i_{q}\left(l_{d}-I_{q}\right),
$$

wobei $\psi_{M}^{\prime}$ der maximale Betrag des Ersatzverkettungsflussraumzeigers im nicht-feldgeschwächten Zustand ist. Im stationären Betriebszustand bei $n=0,1$ und $\underline{i}_{s, d q}=0,5 e^{j 90^{\circ}}$ wurde im nichtfeldgeschwächten Zustand $\gamma_{F W}=0^{\circ}$ ein mittleres Drehmoment von $243 \mathrm{Nm}$ gemessen. Bei einer Halbierung von $\psi_{M}^{\prime}$ mittels $\gamma_{F W}=60^{\circ}$, müsste sich nach (16) auch das Drehmoment halbieren. Tatsächlich gemessen wurden $124 \mathrm{Nm}$, womit die Näherung $m \propto \cos \gamma_{F W}$ hier zulässig ist. Diese Näherung verliert allerdings ihre Gültigkeit, sobald $i_{d} \neq 0$ ist. Wie für einen Rotor mit vergrabenen Permanentmagneten üblich gilt auch hier im Normalzustand $I_{d} / l_{q}<1$ [13]. Durch die Verdrehung der Rotoren im Feldschwächbetrieb ändern sich die Induktivitätsverhältnisse der Maschine, bis schließlich der Bereich $I_{d} / l_{q}>1$ erreicht wird. Das verändert in weiterer Folge auch die maximumtorque-per-current (MTPC) Arbeitspunkte der Maschine und führt dazu, dass die oben genannte Näherung nicht mehr gilt.

\section{Zusammenfassung und Ausblick}

In dieser Arbeit wurde ein neuartiges Konzept einer mechanischen Feldschwächmethode präsentiert, die durch die besondere Struktur des elektrischen Planetenmotors ermöglicht wird. Mit ihr kann 
die induzierte Spannung im laufenden Betrieb durch einen externen Linearaktor verringert werden, um beispielsweise im Fehlerfall im hohen Drehzahlbereich bei offenen Klemmen der Maschine die Spannung auf ein sicheres Niveau zu senken. Mit der am Prototyp implementierten Variante konnte das Funktionsprinzip nachgewiesen werden, wobei die induzierte Spannung bis auf einen geringen hochfrequenten Anteil im stationären Betrieb ausgelöscht werden konnte. Das erhöhte Rastmoment durch die mechanische Feldschwächung sollte für mögliche Anwendungen in der Auslegung der Mechanik, insbesondere des Getriebes, berücksichtigt werden. Die Auswirkung auf die Eisenverluste in der Maschine sowie den Einfluss auf den Wirkungsgrad der Maschine sind Gegenstand zukünftiger Untersuchungen. Weiters sollte für den Einsatz als Fehlerschutzsystem untersucht werden, welche Anforderungen an das transiente Verhalten in realen Anwendungen gestellt werden. Ein möglicher Ansatz zur Verkürzung der Zeit bis zur vollständigen Auslöschung der induzierten Spannung könnte ein vorgespannter Mechanismus mit Federspeichern sein, der eine deutlich schnellere Aktuierung als die bisherige Lösung mit einem Linearmotor ermöglichen würde.

Funding Note Open access funding provided by TU Wien (TUW).

Hinweis des Verlags Der Verlag bleibt in Hinblick auf geografische Zuordnungen und Gebietsbezeichnungen in veröffentlichten Karten und Institutsadressen neutral.

Open Access Dieser Artikel wird unter der Creative Commons Namensnennung 4.0 International Lizenz veröffentlicht, welche die Nutzung, Vervielfältigung, Bearbeitung, Verbreitung und Wiedergabe in jeglichem Medium und Format erlaubt, sofern Sie den/die ursprünglichen Autor(en) und die Quelle ordnungsgemäß nennen, einen Link zur Creative Commons Lizenz beifügen und angeben, ob Änderungen vorgenommen wurden. Die in diesem Artike enthaltenen Bilder und sonstiges Drittmaterial unterliegen ebenfalls der genannten Creative Commons Lizenz, sofern sich aus der Abbildungslegende nichts anderes ergibt. Sofern das betreffende Material nicht unter der genannten Creative Commons Lizenz steht und die betreffende Handlung nicht nach gesetzlichen Vorschriften erlaubt ist, ist für die oben aufgeführten Weiterverwendungen des Materials die Einwilligung des jeweiligen Rechteinhabers ein-

\section{Autoren}

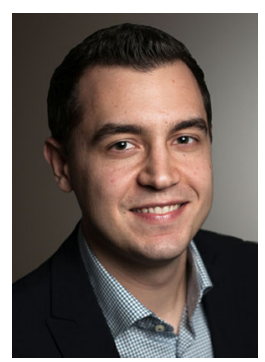

\section{Andreas Brunner}

Studium der Elektrotechnik und Informationstechnik an der Technischen Universität Wien, Österreich, Studienzweig Energie- und Automatisierungstechnik; Abschluss 2018. Seit 2018 Universitätsassistent am Institut für Energiesysteme und Elektrische Antriebe.

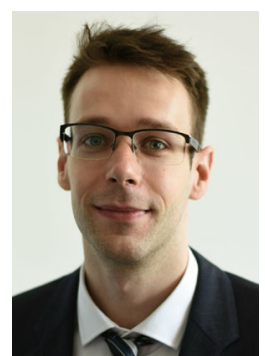

\section{Richard Spießberger}

Studium der Elektrotechnik und Informationstechnik an der Technischen Universität Wien, Österreich, Studienzweig Energie- und Automatisierungstechnik; Abschluss 2018. Seit 2018 Universitätsassistent am Institut für Energiesysteme und Elektrische Antriebe. zuholen. Weitere Details zur Lizenz entnehmen Sie bitte der Lizenzinformation auf http://creativecommons.org/licenses/by/4.0/deed.de.

\section{Literatur}

1. Adnanes, A. K., Nilssen, R., Rad, R. O. (1992): Power feed-back during controller failure in inverter fed permanent magnet synchronous motor drives with flux weakening. In PESC ' 92 record. 23rd annual IEEE power electronics specialists conference (Vol. 2, S. 958-963)

2. Jahns, T. M. (1998): Uncontrolled generator operation of interior PM synchronous machines following high-speed inverter shutdown. In Conference record of 1998 IEEE industry applications conference, Thirty-Third IAS Annual Meeting (Cat. No. 98CH36242) (Vol. 1, S. 395-404)

3. Liaw, C., Soong, W. L., Welchko, B. A., Ertugrul, N. (2005): Uncontrolled generation in interior permanent-magnet machines. IEEE Trans. Ind. Appl., 41, 945-954.

4. Baoquan, K., Chunyan, L., Shukang, C. (2005): A new flux weakening method of permanent magnet synchronous machine. In International conference on electrical machines and systems (Vol. 1, S. 500-503).

5. El-Refaie, A. M. (2011): Fault-tolerant permanent magnet machines: a review. IET Electr. Power Appl., 5(1), 59-74.

6. Woehl-Bruhn, H., Canders, W.-R., Domann, N. (2010): Classification of fieldweakening solutions and novel PM machine with adjustable excitation. In XIX international conference on electrical machines, ICEM 2010 (S. 1-6).

7. Lawrence, P. (2003): Brushless PM motor or alternator with variable axial rotor/stator alignment to increase speed capability. World Intellectual Property Organization: WO 03/077403 A1.

8. Pullen, K. R. (1999): Variable axial airgap rotary electric machines. GB Patent No. 2338117.

9. Rao, D. K. (1999): Axial gap motor with radially movable magnets to increase speed capablity. US Patent No. 6194802B1.

10. Schrödl, M. (2017): Der (elektrische) Planetenmotor - Eine unkonventionelle Kombination von Elektromotor und Planetengetriebe. E\&l, Elektrotech. Inf.tech., 134, 170-176

11. Spiessberger, R., Brunner, A., Schrödl, M. (2019): In The four-pole planetary motor, PCIM Europe 2019, international exhibition and conference for power electronics, intelligent motion, renewable energy and energy management (S. 240-245).

12. Binder, A. (2017): Elektrische Maschinen und Antriebe, Berlin: Springer. ISBN 978-3662-53542-4.

13. Brunner, A., Spiessberger, R., Schrödl, M. (2019): In Sensorless control of a planetary motor, PCIM Europe 2019, international exhibition and conference for power electronics, intelligent motion, renewable energy and energy management (S. 1902-1907).

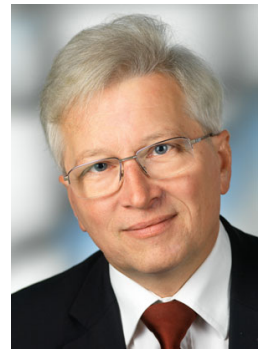

Manfred Schrödl

Studium der Elektrotechnik an der Technischen Universität Wien, Österreich, Studienzweig Industrielle Elektronik und Regelungstechnik; Abschluss 1982. Universitätsassistent am Institut für Elektrische Maschinen und Antriebe der TU Wien, 1987 Promotion zum Dr. techn., 1992 Habilitation für Elektrische Antriebe und Leistungselektronik in der Antriebstechnik. 1993 bis 1996 Leiter F\&E bei Elin Verkehrstechnik Wien, 1996 bis 1998 Bereichsleiter Zentrale Technik bei Flender ATB, Spielberg, Steiermark. Seit 1998 Ordentlicher Universitätsprofessor für Elektrische Antriebe und Maschinen sowie Vorstand des Instituts für Energiesysteme und Elektrische Antriebe an der TU Wien. 